\title{
21. Comparative Karyotypes of Two Land Snails, Euhadra subnimbosa and Euhadra dixoni (Bradybaenidae, Gastropoda)
}

\author{
By Reiko Tatewaki and Jin-ichi Kitada \\ Department of Biology, Shimane Medical University, Izumo, 693 \\ (Communicated by Sajiro Makino, M. J. A., April 12, 1984)
}

Japanese land snails have been classified into about 800 species by Azuma (1982). The chromosome numbers of these animals were studied by Inaba $(1945,1959)$ and Kawabe $(1947)$, based on the classical method with gonadal tissues. They reported the haploid chromosome numbers in 24 species from $n=28$ to $n=29$. Babrakzai and Miller (1975), Babrakzai et al. (1974, 1980) and Reeder and Miller (1974) analyzed the karyotypes of land snails collected from Arizona with the hypotonic squash method. Prasad and Das (1978) first introduced the air-drying technique for the land snail chromosome utilizing their ovo-testes. For the chromosome examination of land snails we had used the chromosome technique reported by Imai et al. (1977) and obtained satisfactory results. Thus our methods were able to get more well-spread metaphase cells than the routine air-drying methods, and the karyotype of two Japanese land snails, E. subnimbosa and E. dixoni, were studied first.

Materials and methods. There are visible differences morphologically in the two species possessing medium sized shells. As described by Azuma (1983) and Inaba (1959), E. subnimbosa belongs to the congenita group, having a remarkable growth line. The shell, not being flattened, is larger and lower than that of $E$. dixoni. This species is distributed in Chugoku, Shikoku and the east coast of Kyushu districts. The twenty adult specimens (0000type) were collected from Onomichi-shi, Hiroshima-ken, in the early spring. While $E$. dixoni is one of the callizona-group, the shell is larger and higher than that of E. callizona; they are distinguished by the genitalia. This species has its habitats in Western Chugoku and Shikoku districts. Thirty individuals of the adult specimens (00(34)type, 02 (34) type) were collected in Matsue-shi, Shimane-ken, before their breeding season.

Snail embryos at ten to twelve days were used for the cytological study. We employed the following chromosome technique of Imai et al. (1977). (1) Incubate the embryos in hypotonic solution for 20-30 min at room temperature and transfer the material onto a 
pre-cleaned slide using a pasteur pipet, (2) drop the $60 \%$ ethanolacetic acid $(3: 1)$ on the slide and crush the material with dissecting needles, (3) add one drop of freshly-prepared absolute ethanol-acetic acid fixative on the slide, (4) allow it to dry completely at room temperature and stain it with Giemsa solution.

Chromosomal morphology assessed from the relative length and the arm ratio was calculated based on 20 well-spread metaphase cells of each species. Nomenclature proposed by Levan et al. (1964) was applied in this study for the karyotype analysis.

Results. The present cytological preparations showed 10-30 well-spread mitotic cells on a slide, in comparison with 0-3 cells following the air-dry methods of Prasad and Das (1978), and that enabled more effective karyotype analysis. With more than 100 metaphasic cells, somatic chromosomes were counted as $2 n=56$ for both species. Those results were identical with Inaba's reports (1945, 1959).

Karyotype analyses were undertaken with 15 well-spread cells of both species (Figs. 1 and 2). Both karyotypes consisted of 29

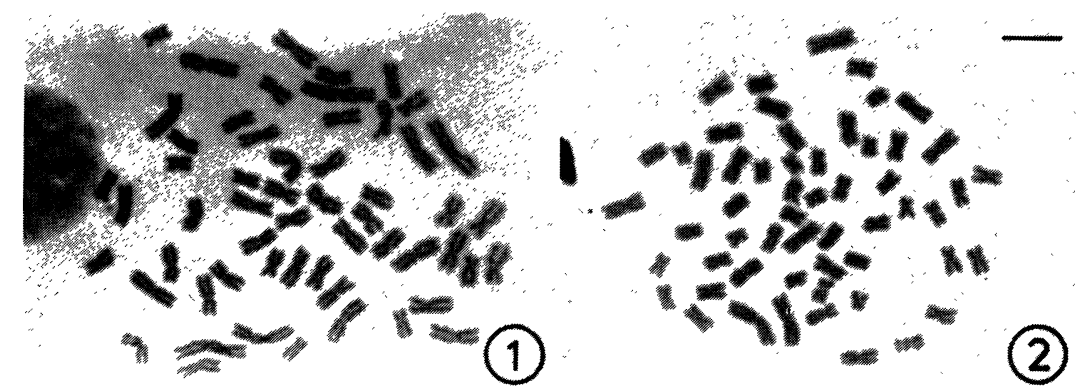

Figs. 1 and 2. Well-spread mitotic cells of Euhadra subnimbosa (1), and Euhadra dixoni (2). Scale: $10 \mu \mathrm{m}$.

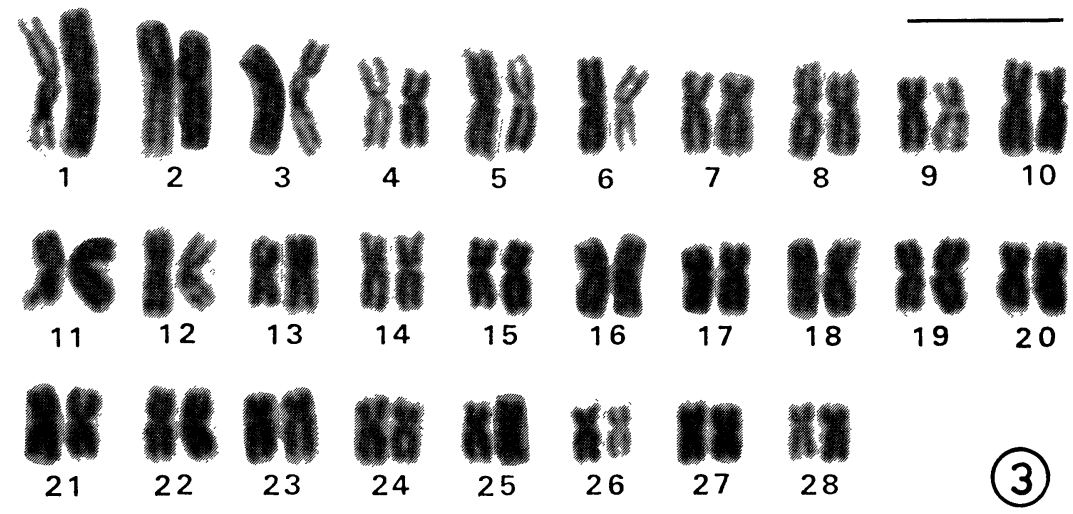

Fig. 3. Karyotype of E. subnimbosa showing 56 chromosomes. Giemsa stain. Scale: $10 \mu \mathrm{m}$. 


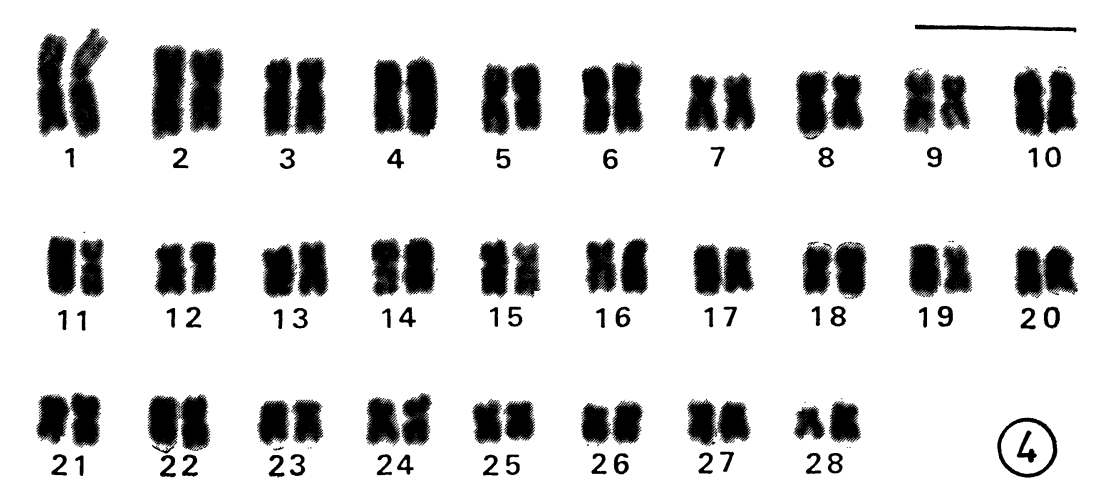

Fig. 4. Karyotype of $E$. dixoni showing 56 chromosomes. Giemsa stain. Scale: $10 \mu \mathrm{m}$.

homologous pairs of metacentric and submetacentric chromosomes as shown in Figs. 3 and 4. E. subnimbosa bears 21 pairs of metacentrics and 7 pairs of submetacentric elements, E. dixoni has 19 pairs of metacentric and 9 pairs of submetacentric chromosomes. On the fundamental number count both showed 112. Furthermore a secondary constriction was detected in the medium sized chromosome pair, no. 14 in E. dixoni, and no. 12 in E. subnimbosa.

Discussion. In six papers describing a technique for land snail chromosomes, two methods have been suggested. One of those is the air-drying technique by Prasad and Das (1978), the other the squash technique by Babrakzai and Miller $(1974,1975)$, Babrakzai et al. (1974, 1976) and Reeder and Miller (1974). From our investigations, however, the present preparations supplied more abundant mitotic cells than the air-drying technique by Prasad and Das (1978). It seems to be prepared without the loss of cells during the procedure.

The results, on examining chromosome number of both species, agreed with Inaba's findings reporting 28 haploid chromosomes for $E$. subnimbosa and 56 chromosomes for $E$. dixoni. These had the same number of chromosomes as that of the majority of species examined in the genus Euhadra (Inaba 1959).

With regard to karyotype analysis in the genus Euhadra, only one paper about $E$. congenita has been published, that by Babrakzai (1980). The species comprised 29 haploid chromosomes consisting of metacentric and submetacentric elements, but E. subnimbosa belonging to the same group in morphology had 28 pairs of metacentric and submetacentric chromosomes, therefore the two species seem to be cytologically different. While a karyotypical difference between subnimbosa and dixoni were observed in the medium sized group as shown in Figs. 3 and 4. E. dixoni bore three submetacentric pairs (nos. $12,15,17$ ), in contrast to E. subnimbosa which contained only 
one pair of submetacentric chromosomes (no. 16). But the remaining large-sized (nos. 1-10) and small-sized (nos. 21-28) chromosomes of both subnimbosa and dixoni were identical, and a secondary constriction was detected in the same sized metacentric pair and in the approximal chromosomal position. In the chromosomal morphology the two species were found to possess similar elements. Furthermore considering that the fundamental number was 112 in each case, both species clearly had a very minor karyotypical difference. This suggests therefore that $E$. subnimbosa and $E$. dixoni are very close in cytological features, although they have obvious differences in morphology.

Summary. Somatic chromosomes of two species in the genus Euhadra were observed using the air-drying methods supplying abundance of mitotic cells. The diploid numbers of $E$. dixoni and E. subnimbosa are confirmed to have 56 in each case. E. subnimbosa bears 21 pairs of metacentric and 7 pairs of submetacentric elements, while $E$. dixoni has 19 pairs of metacentric and 9 pairs of submetacentric chromosomes. They not only show the same fundamental number $\mathrm{NF}=112$, but also have a secondary constriction in the approximal chromosomal position and in the same sized metacentric pairs. Consequently, the minor karyotypical difference found in the two suggests a close similarity cytologically, though they bear morphological differences.

Acknowledgements. We are deeply indebted to Emeritus Professor Sajiro Makino, M. J. A., Hokkaido University, for his critical reading of the manuscript, and to Professor Takeshi Seto, Shimane University, for his helpful advice. We are also very grateful to Mr. Ichiro Okamura, a member of the Malacological Society of Japan, for his useful advice; his identification of the materials used was indispensable for this study.

\section{References}

Azuma, M.: Colored Illustrations of the Land Snails of Japan. Hoikusha, Osaka, pp. 238-300 (in Japanese).

Babrakzai, N., and Miller, W. B. (1974) : Malacol. Review, 7, 37-38.

(1975): Bull. Am. Malacol. Union Inc., 72.

Babrakzai, N. et al. (1974): ibid. 4-11.

Babrakzai, N. et al. (1980) : J. Arizona Nevada Acad. Sci., 15, Proceeding's suppl., 31.

Imai, H. T. et al. (1977) : Chromosoma, 59, 341-393.

Inaba, A. (1945): Venus, 14, 97-102.

- (1959) : J. Sci. Hiroshima Univ., ser. B, 18, 1-29.

Kawabe, M. (1947): La kromosomo, 3-4, 133-134.

Leven, A. et al. (1964): Hereditas, 52, 201-220.

Prasad, R., and Das, C. C. (1978) : The Veliger, 20, 386-387.

Reeder, R., and Miller, B. W. (1974) : Am. Zool., 14, 1264. 